\title{
Imipenem Resistance in Clostridium difficile Ribotype 017, Portugal
}

\section{Joana Isidro, Andrea Santos, Alexandra Nunes, Vítor Borges, Catarina Silva, Luís Vieira, Aristides L. Mendes, Mónica Serrano, Adriano O. Henriques, João Paulo Gomes, Mónica Oleastro}

We describe imipenem-resistant and imipenem-susceptible clinical isolates of Clostridium difficile ribotype 017 in Portugal. All ribotype 017 isolates carried an extra penicillin-binding protein gene, $p b p 5$, and the imipenem-resistant isolates had additional substitutions near the transpeptidase active sites of pbp 1 and pbp3. These clones could disseminate and contribute to imipenem resistance.

Clost lostridium difficile, a toxin-producing, spore-forming bacillus, is a main cause of nosocomial antimicrobial drug-associated diarrhea in industrialized countries (1). C. difficile infection (CDI) usually develops in previously hospitalized persons with a recent history of antimicrobial drug use and causes illness with symptoms ranging from mild diarrhea to potentially lethal pseudomembranous colitis (2). Antimicrobial drugs disrupt the protective gut microbiota, enabling ingested $C$. difficile spores to germinate in the colon and providing a selective advantage to nonsusceptible strains (3). CDI is mainly mediated by the TcdA and TcdB toxins, though some strains additionally produce a binary toxin. Multiple antimicrobial drugs can promote CDI, and cephalosporins and fluoroquinolones have been associated with a higher risk for CDI (3). Multidrug resistance is frequently found in epidemic $C$. difficile strains; determinants of resistance are often found in horizontally transferable mobile genetic elements (4). In past decades, CDI prominence has increased because of a sudden rise in outbreaks and an increase in disease severity and death (5). This shift was mainly associated with the dissemination of fluoroquinolone-resistant PCR ribotype (RT) 027, which has been responsible for hospital outbreaks worldwide. Strains of other ribotypes, including RT078 and RT017, which have enhanced

Author affiliations: National Institute of Health, Lisbon, Portugal (J. Isidro, A. Santos, A. Nunes, V. Borges, C. Silva, L. Vieira, J.P. Gomes, M. Oleastro); Instituto de Tecnologia Química e Biológica António Xavier, Oeiras, Portugal (A.L. Mendes, M. Serrano, A.O. Henriques)

DOI: https://doi.org/10.3201/eid2404.170095 virulence, have emerged (6). In particular, RT017, the most common toxin A-negative, toxin $\mathrm{B}$-positive ribotype, is widespread in Asia and is common in Europe (79 ). In a pan-European study of $\approx 900 C$. difficile strains, the overall rate of resistance to imipenem, an antimicrobial drug of the carbapenem class, currently widely used as a last-line drug to treat infections by gram-negative bacteria, was found to be $7.41 \%$, and the geometric mean (GM) MIC of imipenem for RT017 strains was $5.91 \mathrm{mg} / \mathrm{L}(8)$. In another study, isolates collected in a South Korea hospital during 2000-2009 were analyzed, and a resistance rate to imipenem of $8 \%$ ( $12 \%$ among RT017 isolates) was found (10).

\section{The Study}

We characterized $191 C$. difficile isolates collected during September 2012-September 2015 from 15 hospitals in Portugal (online Technical Appendix, https://wwwnc.cdc. gov/EID/article/24/4/17-0095-Techapp1.pdf). We found $24(12.6 \%)$ were resistant to imipenem. Of these 24 isolates, 22 were RT017, 1 was RT014, and 1 was RT477. The MIC for imipenem for RT017, the imipenem-resistant isolates, was $>32 \mathrm{mg} / \mathrm{L}$ (Table 1); the MIC for the 2 nonRT017 isolates was $16 \mathrm{mg} / \mathrm{L}$. The 22 imipenem-resistant RT017 isolates were found at hospital A throughout the study period, suggesting the existence of a persistent clone, a finding supported by whole-genome sequencing data (online Technical Appendix). Among the 25 RT017 isolates, 3 were imipenem-susceptible and from hospital B (MIC range $1.5-3 \mathrm{mg} / \mathrm{L}$ ) (Table 1$)$.

RT017 C. difficile strains are frequently resistant to clindamycin, erythromycin, moxifloxacin, tetracycline, or rifampin (individually or in combination) $(8,10)$. In this study, the 22 RT017 imipenem-resistant isolates were also found to be resistant to all of these antimicrobial drugs and showed higher meropenem and ertapenem MICs than those of the RT017 imipenem-susceptible isolates (Table 1; online Technical Appendix; online Technical Appendix Figure). Multidrug resistance to noncarbapenem antimicrobial drugs correlated with the presence of several genetic determinants, many located in mobile genetic elements (Figure 1; online Technical Appendix), in line with the idea that multidrug-resistant strains have a selective advantage (4) and that horizontal gene transfer plays a major role in the evolution of this pathogen (11). 
Table 1. Susceptibility of Clostridium difficile RT017 imipenem-resistant isolates from hospital A and imipenem-susceptible isolates from hospital B to 11 antimicrobial drugs, Portugal*

\begin{tabular}{|c|c|c|c|c|c|c|c|c|c|c|c|c|}
\hline \multirow[b]{3}{*}{ Hospital } & \multirow{3}{*}{$\begin{array}{l}\text { Resistance } \\
\text { breakpoint }\end{array}$} & \multicolumn{11}{|c|}{ Antimicrobial drug, MIC breakpoints, mg/L } \\
\hline & & IMP & ETP & MRP $\ddagger$ & MXF+§ & MTZ†§ & VAN†§ & CLI† & CHL‡ & RIF† & TGC† & TET \\
\hline & & $\geq 16$ & $\geq 16$ & $\geq 16$ & $>4$ & $>2$ & $>2$ & $\geq 8$ & $\geq 32$ & $>0.004$ & $>0.25$ & $\geq 16$ \\
\hline \multirow{5}{*}{$\begin{array}{l}\mathrm{A}, 22 \\
\text { isolates }\end{array}$} & MIC range & $>32$ & $\overline{3-16}$ & $1.5-4$ & $>32$ & $<0.016-1$ & $0.38-2$ & $>\overline{256}$ & $\overline{2}-6$ & $>32$ & $<0.016-0.094$ & $\overline{16}-32$ \\
\hline & GM MIC & 32 & 7.56 & 2.31 & 32 & 0.12 & 0.73 & 256 & 3.29 & 32 & 0.025 & 18.08 \\
\hline & $\mathrm{MIC}_{90}$ & 32 & 12 & 3 & $>32$ & 0.38 & 2 & 256 & 4 & 32 & 0.032 & 32 \\
\hline & $\mathrm{MIC}_{50}$ & 32 & 6 & 2 & $>32$ & 0.19 & 0.75 & 256 & 3 & 32 & 0.023 & 16 \\
\hline & $\%$ Resistant & 100 & 4.5 & 0 & 100 & 0 & 0 & 100 & 0 & 100 & 0 & 100 \\
\hline & MIC range & $1.5-3$ & $1.5-2$ & $0.5-1.5$ & 1.5 & $<0.016-0.25$ & $0.38-0.75$ & $>256$ & $3-4$ & $>32$ & $<0.016-0.023$ & 16 \\
\hline \multirow{4}{*}{ isolates } & GM MIC & 2.08 & 1.82 & 0.83 & 1.5 & 0.072 & 0.60 & 256 & 3.30 & 32 & 0.020 & 16 \\
\hline & $\mathrm{MIC}_{90}$ & 3 & 2 & 1.5 & 1.5 & 0.25 & 0.75 & 256 & 4 & 32 & 0.023 & 16 \\
\hline & $\mathrm{MIC}_{50}$ & 2 & 2 & 0.75 & 1.5 & 0.094 & 0.75 & 256 & 3 & 32 & 0.023 & 16 \\
\hline & $\%$ Resistant & 0 & $\overline{0}$ & 0 & 0 & 0 & 0 & 100 & 0 & 100 & 0 & 100 \\
\hline$p$ value & & $<0.0001$ & $<0.0001$ & $<0.0001$ & $<0.0001$ & 0.45 & 0.56 & ND & 0.98 & ND & 0.41 & 0.51 \\
\hline \multicolumn{13}{|c|}{$\begin{array}{l}\text { }{ }^{*} \mathrm{CHL} \text {, chloramphenicol; CLI, clindamycin; ETP, ertapenem; GM, geometric mean; IMP, imipenem; MIC } 50 \text {, minimal inhibitory concentration for } 50 \% \text { of } \\
\text { strains; MIC } \text { I }_{90} \text {, minimal inhibitory concentration for } 90 \% \text { of strains; MRP, meropenem; MTZ, metronidazole; MXF, moxifloxacin; ND, not done; RIF, } \\
\text { rifampin; TGC, tigecycline; VAN, vancomycin. } \\
\text { †European Committee on Antimicrobial Susceptibility Testing breakpoint. } \\
\text { †Clinical and Laboratory Standards Institute breakpoint. } \\
\text { §Previously determined (9). }\end{array}$} \\
\hline
\end{tabular}

Through whole-genome sequencing, we found 13 single-nucleotide polymorphisms (SNPs) that differentiated the imipenem-resistant and imipenem-susceptible RT017 isolates (Table 2; Figure 1; online Technical Appendix). We found 2 SNPs in the genes coding for 2 high molecular weight (HMW) penicillin-binding proteins (PBPs) (Figure 1). HMW PBPs, which are bifunctional enzymes containing transglycosylase and transpeptidase domains, are categorized into class A, and PBPs lacking the transglycosylase domain are categorized into class B. The transpeptidase domain harbors 3 functional motifs (SXXK, SXN, and $\mathrm{KTG}[\mathrm{T} / \mathrm{S}]$ ) that comprise the active site. Carbapenems block cell wall synthesis by inhibiting transpeptidase activity (12). One of the mutations found in the imipenem-resistant isolates affected the gene coding for PBP1, the single class A bifunctional peptidoglycan synthase of $C$. difficile; the mutation resulted in the amino acid substitution Ala555Thr close to the SSN functional motif (Figure 2). The second mutation was found in the gene encoding for the PBP3 class B transpeptidase and caused the amino acid replacement Tyr721Ser between the SXN and KTGT motifs (Figure 2). Neither of these changes was found in the 3 imipenem-susceptible RT017 isolates (Figure 1). Moreover, the 2 non-RT017 imipenem-resistant isolates, with a MIC for imipenem lower than that of the RT017 isolates, revealed either the Ala555Thr change or a different substitution (Leu543His), both in PBP1, also close to the functional motif SXN (Table 2). Modified PBPs with reduced affinity for the antimicrobial drug have been associated with resistance to $\beta$-lactams and specifically to imipenem in several microorganisms (12). We found no differences between the imipenem-resistant and imipenem-susceptible RT017 isolates in genes encoding other peptidoglycan synthases (Figure 1). It is possible that the substitutions in PBP1 and PBP3 in RT017 confer high-level resistance to imipenem and reduced susceptibility to other carbapenems, and at least in the RT014 and RT477 isolates studied, the single Ala555Thr substitution (or other substitutions in the vicinity of the SXN motif) is sufficient for an intermediate level of resistance.

However, all RT017 isolates studied herein, as well as the previously annotated strains M68 (GenBank accession no. NC_017175) and BJ08 (accession no. CP003939), have a fifth HMW class B PBP, PBP5, encoded in a mobile element (online Technical Appendix). Whether PBP5 contributes to imipenem resistance remains to be determined. Moreover, in imipenem-resistant isolates, the key sporulation-specific gene $\operatorname{sig} K$, which is contiguous to $p b p 2$, is interrupted by the 17-kb skin cd element (13), and the $p b p 5$ region is contiguous to a transposon-like element carrying the ermB gene (shown as PUBMLST allele 8; https://pubmlst.org/bigsdb?db=pubmlst_cdifficile_se qdef\&page $=$ alleleInfo\&locus $=$ ermB\&allele $\_$id $=8$ ). It is unknown whether these genetic differences contribute to imipenem resistance.

\section{Conclusions}

Imipenem resistance in $C$. difficile RT017 probably involves the acquisition of mutations in both $p b p 1$ and $p b p 3$ that lead to amino acid substitutions close to the functional motifs of their transpeptidase domains. These substitutions might decrease the affinity of PBP1 and PBP3 for imipenem, enabling peptidoglycan synthesis in the presence of the antimicrobial drug. Considering that the presence of an additional PBP (PBP5) is a characteristic of RT017 strains, we suggest that PBP5 facilitates the expression of imipenem resistance through acquisition of mutations in $p b p 1$ and $p b p 3$. In strains of other ribotypes lacking PBP5, such as the RT014 and RT477 isolates herein described, mutations in $p b p 1$ might only lead to 


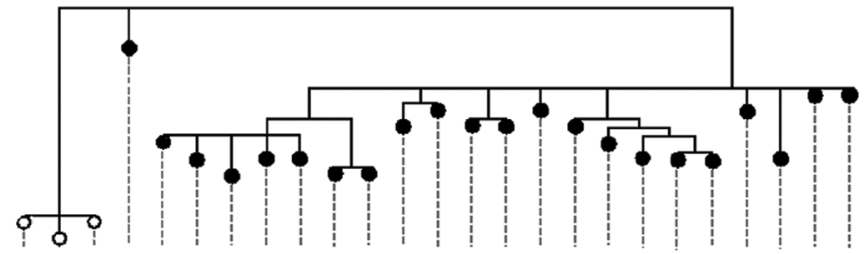

Resistant to imipenem

O Susceptible to impenem

B1 B2 B3 A1 A2 A16 A8 A7 A4 A19A15 A5 A17 A6 A20A11 A3 A13 A9 A21 A22 A10A18 A12 A14

Gene/nt change/aa change Antibiotic

D

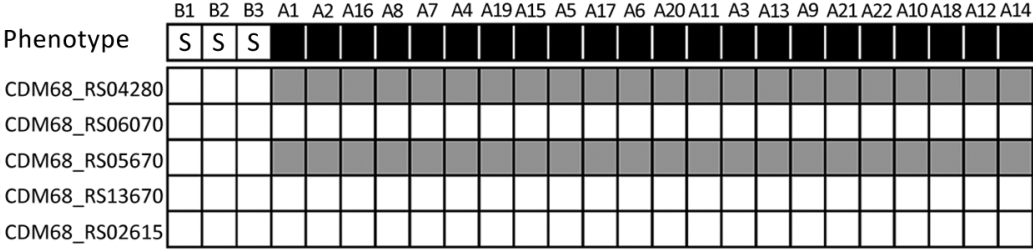

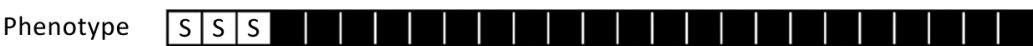

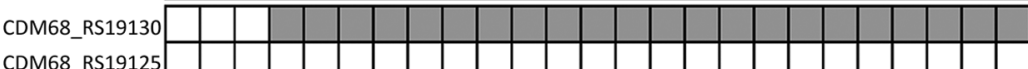

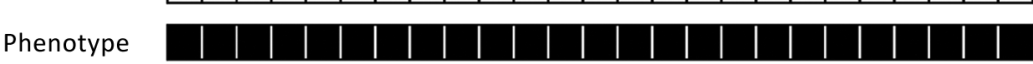

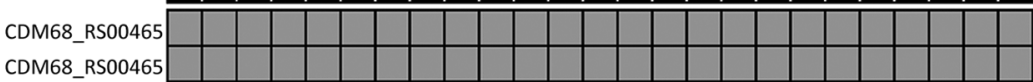

pbp1/G1663A/Ala555Thr
pbp2 (no mutations)
pbp3/A2162C/Tyr721Ser
pbp4 (no mutations)
pbp5 (no mutations)

Carbapenems

Imipenem
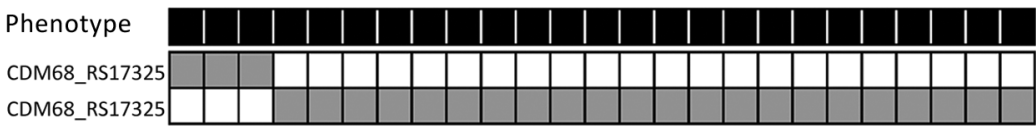

CDM68_RS17325

pbps

\begin{tabular}{|c|c|}
\hline & Fluoroquinolone \\
\hline $\begin{array}{l}\text { gyrA/C245T/Thr82lle } \\
\text { gyrB (no mutations) }\end{array}$ & Moxifloxacin \\
\hline
\end{tabular}

\section{gre (no mutations) \\ rpoB/C1504A/His502Asn rpoB/G1514A/Arg505Lys}

Rifampin
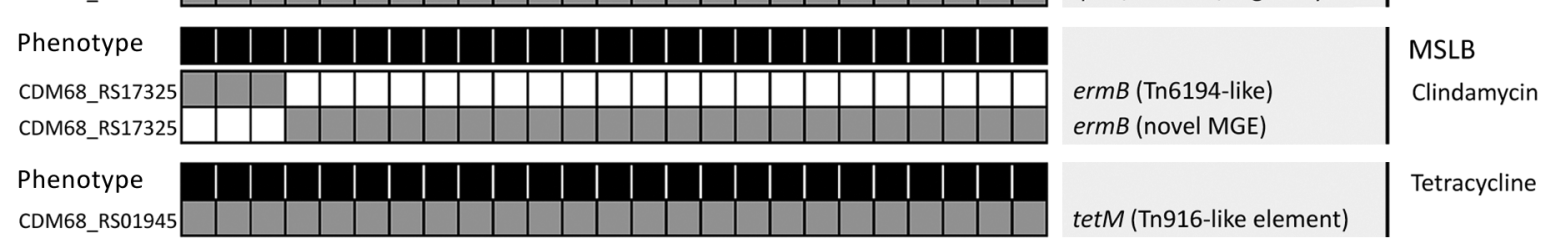

tetM (Tn916-like element)

Tetracycline

$$
\begin{array}{ll}
\text { Phenotype } & \text { Gene or mutation } \\
\hline \text { S } & \begin{array}{l}
\text { Resistant } \\
\text { Susceptible }
\end{array}
\end{array}
$$

Figure 1. Phylogeny of Clostridium difficile RT017 isolates from hospitals A and B and genetic determinants of antimicrobial drug resistance, Portugal. A) Core genome single-nucleotide polymorphism-based neighbor-joining phylogeny of 25 RT017 C. difficile clinical isolates reconstructed by using 47 variant sites (outside MGEs) identified when mapping to either the corresponding genomic sequence of close relative C. difficile strain M68 (GenBank accession no. NC_017175) or a draft genome sequence of a representative clinical isolate. B) For each isolate, the profile of antimicrobial drug susceptibility is indicated together with respective potential genetic determinants of antimicrobial drug resistance. Only antimicrobial drugs for which a resistant phenotype was observed are displayed. Gene locus tags are relative to the $C$. difficile M68 genome annotation. Both nucleotide and amino acid replacements refer to mutations in the resistant isolates when comparing with susceptible isolates. No mutations means that no mutations are present differentiating resistant isolates of hospital A from susceptible isolates of hospital $\mathrm{B}$, although mutations are present relative to $\mathrm{M} 68$. Both the pbp5-carrying region and the ermB gene (present in all isolates) were found to be inserted in distinct genomic contexts (online Technical Appendix, https://wwwnc.cdc.gov/EID/article/24/4/17-0095-Techapp1.pdf). MSLB, macrolide/ lincosamide/streptogramin B; MGE, mobile genetic element.

intermediate levels of resistance. We further suggest that the spreading of $p b p 5$ might contribute to the dissemination of high-level imipenem resistance.

Portugal has a high rate of healthcare-associated infections and is a major consumer of carbapenems (1). Although carbapenem consumption has not been directly linked to $C$. difficile resistance, we speculate that the emergence of resistance and reduced susceptibility to these antimicrobial drugs might recapitulate the scenario observed with fluoroquinolone-resistant RT027 in the United States, where fluoroquinolones were the most prescribed antimicrobial drug (14). Our findings further reinforce the need for the responsible use of antimicrobial drugs; the emergence of carbapenem resistance in multidrug-resistant $C$. difficile clones might result in the dissemination of resistant strains.

\section{Acknowledgments}

We thank the participating hospitals for providing samples for the laboratory surveillance of CDI.

This work was supported by the National Institute of Health Dr. Ricardo Jorge (grant no. 2016DDI1284). We also acknowledge grant Pest-C/EQB/LA0006/2011 from Fundação para a Ciência e a Tecnologia (FCT; http://www.fct.pt) and program Investigador FCT (IF/00268/2013/CP1173/CT0006 to M.S.). A.L.M. was supported by a PhD fellowship (PD/BD/105738/2014) from the FCT. 
Table 2. Mutations differentiating Clostridium difficile RT017 imipenem-resistant isolates found at hospital A from imipenemsusceptible isolates found at hospital B, Portugal

\begin{tabular}{|c|c|c|c|c|c|}
\hline $\begin{array}{l}\text { Gene in M68 } \\
\text { genome* }\end{array}$ & $\begin{array}{l}\text { Genome } \\
\text { position* }\end{array}$ & $\begin{array}{l}\text { Nucleotide } \\
\text { in M68 }\end{array}$ & $\begin{array}{l}\text { Nucleotide } \\
\text { change }\end{array}$ & Amino acid change $†$ & Gene product \\
\hline RS02665 & 512416 & $\mathrm{C}$ & $\mathrm{C} 578 \mathrm{~T}$ & Ala193Val $\ddagger$ & $\begin{array}{l}\text { Multidrug ATP-binding cassette transporter permease, } \\
\text { associated with antimicrobial drug resistance }\end{array}$ \\
\hline RS04280/pbp1 & 905394 & G & G1663A & Ala555Thr & Penicillin-binding transpeptidase \\
\hline RS04935 & 1048151 & C & T1010C & Ile337Thr‡ & 3-Isopropylmalate dehydratase large subunit \\
\hline RS05670/pbp3 & 1221182 & G & A2162C & Tyr721Ser‡ & Penicillin-binding protein \\
\hline RS07765 & 1666351 & G & G214T & Gly72§ & Hypothetical protein \\
\hline RS07795/hisB & 1671129 & $\mathrm{~T}$ & T209C & lle70Thr & Imidazoleglycerol-phosphate dehydratase \\
\hline RS07810 & 1673280 & $\mathrm{~T}$ & C474T & Ala158Ala & Imidazoleglycerol-phosphate synthase cyclase subunit \\
\hline RS08415 & 1792079 & G & A241G & Lys81Gluł & $\begin{array}{c}\text { Hypothetical protein (domain of MerR-like } \\
\text { transcriptional regulators) }\end{array}$ \\
\hline RS08810 & 1882950 & C & C420T & Asp140Asp & Flavodoxin \\
\hline RS14235 & 3083548 & G & G421T & Gly141§ & Haloacid dehalogenase \\
\hline RS18530 & 4054525 & C & C220T & GIn74§ & S-adenosyl methionine-dependent methyltransferase \\
\hline RS19130/gyrA & 4174650 & C & $\mathrm{C} 245 \mathrm{~T}$ & Thr82lle $\ddagger$ & DNA gyrase subunit $A$ \\
\hline RS19545 & 4255124 & $\mathrm{C}$ & C400T & His134Tyrł & Phage portal, SPP1 Gp6-like family protein \\
\hline
\end{tabular}

A

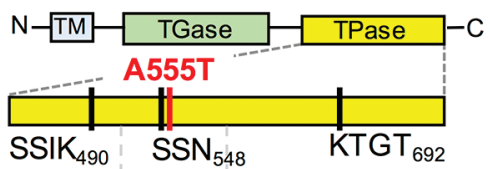

PBP1

A555T

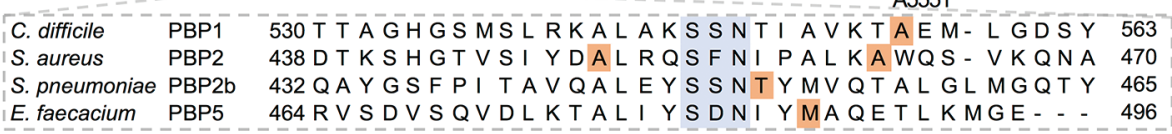

A555T T446A M485A A450D

B

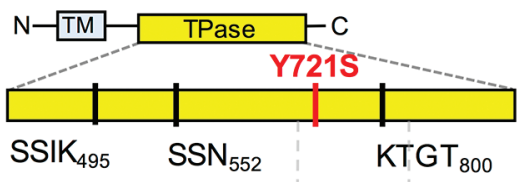

PBP3

$Y 721 \bar{S}$

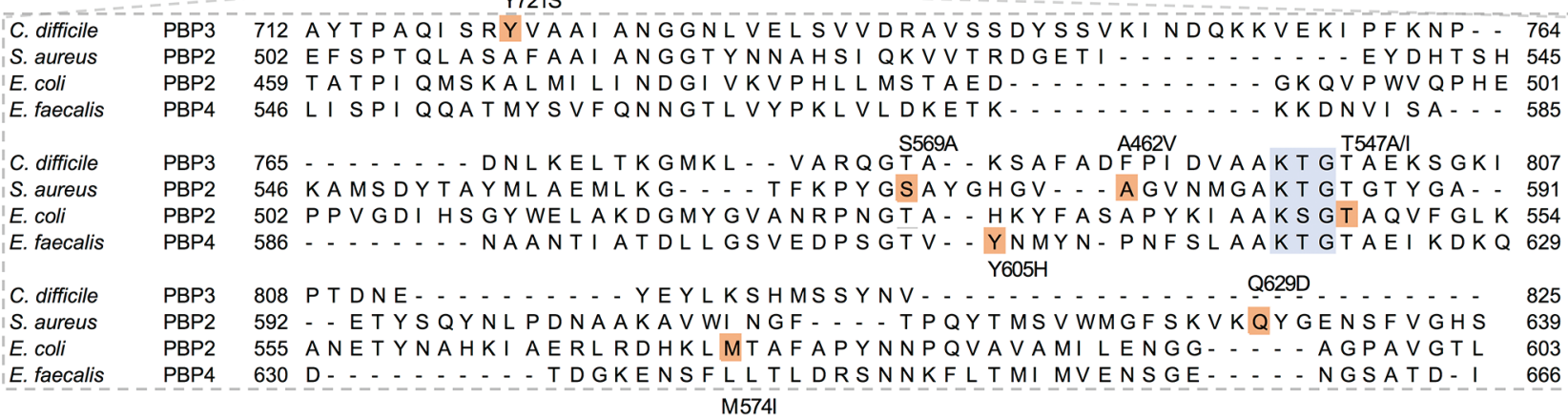

Figure 2. Amino acid substitutions in 2 PBPs predicted to be associated with imipenem resistance in Clostridium difficile, Portugal.

The domains and conserved motifs SXXK, SXN, and KTG[T/S] are shown for the following proteins: PBP1 (A), homolog of CDM68_RS04280 of RT017 strain M68 (GenBank accession no. NC_017175) or CD630_07810 in the laboratory strain 630; and PBP3

(B), homolog of CDM68_RS05670 or CD630_11480. The mutations found in these resistant isolates are marked by red lines. The alignments below the 2 proteins show the position (shaded in pink) and nature of the amino acid substitutions observed in the imipenemresistant RT017 isolates and select PBPs from microorganisms Staphylococcus aureus (GenBank accession no. AAA74375.1), Streptococcus pneumoniae (GenBank accession no. WP_001829432.1), Escherichia coli (GenBank accession no. AAB40835.1), Enterococcus faecalis (GenBank accession no. AAS77615.1), and Enterococcus faecacium (GenBank accession no. AIG13039.1). The conserved motifs in the vicinity of the substitutions are shaded in blue. PBP, penicillin-binding protein; TGase, transglycosylase; TM, transmembrane; TPase, transpeptidase. 


\section{About the Author}

Dr. Isidro is a fellow in the Reference Laboratory for Gastrointestinal Infections at the National Institute of Health Dr. Ricardo Jorge in Lisbon, Portugal. Her primary research interests include CDI, antibiotic resistance, molecular typing, and wholegenome sequencing.

\section{References}

1. European Centre for Disease Prevention and Control. Point prevalence survey of healthcare-associated infections and antimicrobial use in European acute care hospitals, 2011-2012. Stockholm: The Centre; 2013.

2. Smits WK, Lyras D, Lacy DB, Wilcox MH, Kuijper EJ. Clostridium difficile infection. Nat Rev Dis Primers. 2016;2:201620.

3. Slimings C, Riley TV. Antibiotics and hospital-acquired Clostridium difficile infection: update of systematic review and meta-analysis. J Antimicrob Chemother. 2014;69:881-91. http://dx.doi.org/10.1093/jac/dkt477

4. Spigaglia P. Recent advances in the understanding of antibiotic resistance in Clostridium difficile infection. Ther Adv Infect Dis. 2016;3:23-42. http://dx.doi.org/10.1177/2049936115622891

5. Gerding DN, Lessa FC. The epidemiology of Clostridium difficile infection inside and outside health care institutions. Infect Dis Clin North Am. 2015;29:37-50. http://dx.doi.org/ 10.1016/j.idc.2014.11.004

6. Freeman J, Bauer MP, Baines SD, Corver J, Fawley WN, Goorhuis B, et al. The changing epidemiology of Clostridium difficile infections. Clin Microbiol Rev. 2010;23:529-49. http://dx.doi.org/10.1128/CMR.00082-09

7. King AM, Mackin KE, Lyras D. Emergence of toxin A-negative, toxin B-positive Clostridium difficile strains: epidemiological and clinical considerations. Future Microbiol. 2015;10:1-4. http://dx.doi.org/10.2217/fmb.14.115

8. Freeman J, Vernon J, Morris K, Nicholson S, Todhunter S, Longshaw C, et al.; Pan-European Longitudinal Surveillance of
Antibiotic Resistance among Prevalent Clostridium difficile Ribotypes' Study Group. Pan-European longitudinal surveillance of antibiotic resistance among prevalent Clostridium difficile ribotypes. Clin Microbiol Infect. 2015;21:248.e9-16. http://dx.doi.org/10.1016/j.cmi.2014.09.017

9. Santos A, Isidro J, Silva C, Boaventura L, Diogo J, Faustino A, et al. Molecular and epidemiologic study of Clostridium difficile reveals unusual heterogeneity in clinical strains circulating in different regions in Portugal. Clin Microbiol Infect. 2016;22:695700. http://dx.doi.org/10.1016/j.cmi.2016.04.002

10. Lee JH, Lee Y, Lee K, Riley TV, Kim H. The changes of PCR ribotype and antimicrobial resistance of Clostridium difficile in a tertiary care hospital over 10 years. J Med Microbiol. 2014;63:81923. http://dx.doi.org/10.1099/jmm.0.072082-0

11. He M, Sebaihia M, Lawley TD, Stabler RA, Dawson LF, Martin MJ, et al. Evolutionary dynamics of Clostridium difficile over short and long time scales. Proc Natl Acad Sci U S A. 2010;107:7527-32. http://dx.doi.org/10.1073/pnas.0914322107

12. Zapun A, Contreras-Martel C, Vernet T. Penicillin-binding proteins and $\beta$-lactam resistance. FEMS Microbiol Rev. 2008;32:361-85. http://dx.doi.org/10.1111/j.1574-6976.2007.00095.x

13. Serrano M, Kint N, Pereira FC, Saujet L, Boudry P, Dupuy B, et al. A recombination directionality factor controls the cell type-specific activation of $\sigma^{\mathrm{K}}$ and the fidelity of spore development in Clostridium difficile. PLoS Genet. 2016;12:e1006312. http://dx.doi.org/10.1371/journal.pgen.1006312

14. He M, Miyajima F, Roberts P, Ellison L, Pickard DJ, Martin MJ, et al. Emergence and global spread of epidemic healthcareassociated Clostridium difficile. Nat Genet. 2013;45:109-13. http://dx.doi.org/10.1038/ng.2478

Address for correspondence: Mónica Oleastro, National Institute of Health Dr. Ricardo Jorge, National Reference Laboratory for Gastrointestinal Infections, Department of Infectious Diseases, Av Padre Cruz, 1649-016 Lisbon, Portugal; email: monica.oleastro@insa.min-saude.pt

\title{
PubMed Central
}

\section{PublMed}

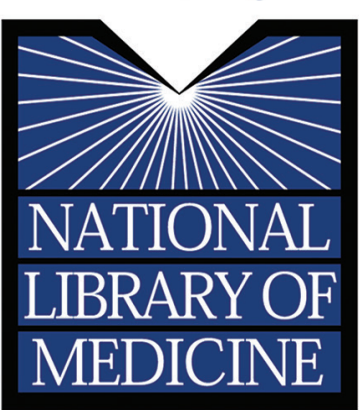

\section{Find Emerging Infectious Diseases content in the digital archives of the National Library of Medicine}

\author{
www.pubmedcentral.nih.gov
}

